\title{
Pontryagin Maximum Principle for Distributed-Order Fractional Systems
}

\author{
Faïçal Ndaïrou ${ }^{t, \ddagger(1)}$ and Delfim F. M. Torres * \\ Center for Research and Development in Mathematics and Applications (CIDMA), Department of Mathematics, \\ University of Aveiro, 3810-193 Aveiro, Portugal; faical@ua.pt \\ * Correspondence: delfim@ua.pt; Tel.: +351-234-370-668 \\ + This research is part of first author's Ph.D. project, which is carried out at the University of Aveiro under the \\ Doctoral Program in Applied Mathematics of Universities of Minho, Aveiro, and Porto (MAP-PDMA). \\ $\ddagger$ These authors contributed equally to this work.
}

check for

updates

Citation: Ndaïrou, F.; Torres, D.F.M. Pontryagin Maximum Principle for Distributed-Order Fractional Systems. Mathematics 2021, 9, 1883. https:// doi.org/10.3390/math9161883

Academic Editor: Eva Kaslik

Received: 2 July 2021

Accepted: 6 August 2021

Published: 8 August 2021

Publisher's Note: MDPI stays neutral with regard to jurisdictional claims in published maps and institutional affiliations.

Copyright: (c) 2021 by the authors. Licensee MDPI, Basel, Switzerland. This article is an open access article distributed under the terms and conditions of the Creative Commons Attribution (CC BY) license (https:// creativecommons.org/licenses/by/ $4.0 /)$.
Abstract: We consider distributed-order non-local fractional optimal control problems with controls taking values on a closed set and prove a strong necessary optimality condition of Pontryagin type. The possibility that admissible controls are subject to pointwise constraints is new and requires more sophisticated techniques to include a maximality condition. We start by proving results on continuity of solutions due to needle-like control perturbations. Then, we derive a differentiability result on the state solutions with respect to the perturbed trajectories. We end by stating and proving the Pontryagin maximum principle for distributed-order fractional optimal control problems, illustrating its applicability with an example.

Keywords: distributed-order fractional calculus; optimal control; Pontryagin maximum principle; needle-like variations

MSC: 26A33; 49K15

\section{Introduction}

The idea to consider fractional order systems of distributed order goes back to Caputo and the study of anomalous diffusion in viscoelasticity [1]. The interest on the new operator slowly increased, in particular with the works of Chechkin et al. [2], who applied distributed order fractional derivatives to study retarding sub-diffusion and accelerating super-diffusion; Naber [3] studied distributed-order fractional subdiffusion processes with different decay rates; Kochubei [4] applied distributed-order operators to the study of ultraslow diffusion; and Mainardi et al. [5] applied distributed order fractional derivatives to study Gaussian diffusion. The subject is today under strong current research, partially explained by their relation with physical processes lacking temporal scaling [6] and complex non-linear systems [7]. Indeed, the distributed-order definition of the operator allows considering superposition of orders and accounting for physical phenomena, such as memory effects in composite materials and multi-scale effects. A typical example that illustrates the capabilities of this class of operators is the mechanical behavior of viscoelastic materials having spatially varying properties. The literature on experimental applications of fractional order systems of distributed order is now vast, and we refer the interested reader to the review paper of Reference [8]. For numerical aspects of fractional initial value problems of distributed-order, we refer to Reference [9].

The calculus of variations is a field of mathematical analysis that uses variations, which are small perturbations in functions to find maxima and minima of functionals. The Euler-Lagrange equation is the main tool for solving such optimization problems, and they have been developed in the context of fractional calculus to better describe non-conservative systems in mechanics [10]. Necessary optimality condition of EulerLagrange type for distributed-order problems of the calculus of variations were first 
introduced and developed in Reference [11]. The results were then further generalized by the present authors in Reference [12], with the proof of several analytical results and a weak maximum principle of Pontryagin type for distributed-order fractional optimal control problems. Here, we extend and improve the theory of optimal control for distributed-order fractional operators initiated in Reference [12] by proving a strong version of the Pontryagin maximum principle, which allows the values of the controls to be constrained to a closed set. The main novelty consists to extend the optimality condition proved in Reference [12] to a maximality condition, which yields to the strong version of Pontryagin maximum principle. For this purpose, and in contrast with Reference [11,12], we use the so-called needle-like variations to the control perturbations.

The paper is organized as follows. In Section 2, we recall some necessary results of the distributed-order fractional calculus. Our contribution is given in Section 3: we formulate the distributed-order fractional optimal control problem under investigation, and we prove the continuity of solutions (Lemmas 3 and 4), a result on the differentiability of the perturbed trajectories (Lemma 5) and, finally, the Pontryagin maximum principle (Theorem 1). We then give an illustrative example of application of the obtained necessary optimality conditions in Section 4. We end with Section 5, indicating some conclusions, the main achievements and novelty of the work, as well as some future research directions.

\section{Preliminaries}

In this section, we recall necessary results and fix notations. We assume the reader to be familiar with the standard Riemann-Liouville and Caputo fractional calculi $[13,14]$.

Let $\alpha$ be a real number in $[0,1]$. In the sequel, we use the following notation:

$$
L^{\alpha}\left([a, b], \mathbb{R}^{n}\right):=\left\{x \in L^{1}\left([a, b], \mathbb{R}^{n}\right): I_{a^{+}}^{\alpha} x, I_{b^{-}}^{\alpha} x \in A C\left([a, b], \mathbb{R}^{n}\right)\right\},
$$

where $I_{a^{+}}^{\alpha}$ and $I_{b^{-}}^{\alpha}$ represent, respectively, the left and right Riemann-Liouville integral of order $\alpha$. We also use the notation $A C^{\alpha}\left([a, b], \mathbb{R}^{n}\right)$ to represent the set of absolutely continuous functions that can be represented as

$$
x(t)=x(a)+I_{a^{+}}^{\alpha} f(t) \quad \text { and } \quad x(t)=x(b)+I_{b^{-}}^{\alpha} f(t),
$$

for some functions $f \in L^{\alpha}$.

Let $\psi$ be a non-negative continuous function defined on $[0,1]$ such that

$$
\int_{0}^{1} \psi(\alpha) d \alpha>0
$$

This function $\psi$ will act as a distribution of the order of differentiation.

Definition 1 (See Reference [15]). The left- and right-sided Riemann-Liouville distributed-order fractional derivatives of a function $x \in L^{\alpha}$ are defined, respectively, by

$$
\mathbb{D}_{a^{+}}^{\psi(\cdot)} x(t)=\int_{0}^{1} \psi(\alpha) \cdot D_{a^{+}}^{\alpha} x(t) d \alpha \quad \text { and } \quad \mathbb{D}_{b^{-}}^{\psi(\cdot)} x(t)=\int_{0}^{1} \psi(\alpha) \cdot D_{b^{-}}^{\alpha} x(t) d \alpha,
$$

where $D_{a^{+}}^{\alpha}$ and $D_{b^{-}}^{\alpha}$ are, respectively, the left- and right-sided Riemann-Liouville fractional derivatives of order $\alpha$.

Definition 2 (See Reference [15]). The left-and right-sided Caputo distributed-order fractional derivatives of a function $x \in A C^{\alpha}$ are defined, respectively, by

$$
{ }^{C} \mathbb{D}_{a^{+}}^{\psi(\cdot)} x(t)=\int_{0}^{1} \psi(\alpha) \cdot{ }^{C} D_{a^{+}}^{\alpha} x(t) d \alpha \quad \text { and } \quad{ }^{C} \mathbb{D}_{b^{-}}^{\psi(\cdot)} x(t)=\int_{0}^{1} \psi(\alpha) \cdot{ }^{C} D_{b^{-}}^{\alpha} x(t) d \alpha,
$$


where ${ }^{C} D_{a^{+}}^{\alpha}$ and ${ }^{C} D_{b^{-}}^{\alpha}$ are, respectively, the left- and right-sided Caputo fractional derivatives of $\operatorname{order} \alpha$.

As noted in Reference [11], there is a relation between the Riemann-Liouville and the Caputo distributed-order fractional derivatives:

$$
\mathrm{C}_{\mathbb{D}_{a^{+}}^{\psi(\cdot)}} x(t)=\mathbb{D}_{a^{+}}^{\psi(\cdot)} x(t)-x(a) \int_{0}^{1} \frac{\psi(\alpha)}{\Gamma(1-\alpha)}(t-a)^{-\alpha} d \alpha
$$

and

$$
{ }^{C} \mathbb{D}_{b^{-}}^{\psi(\cdot)} x(t)=\mathbb{D}_{b^{-}}^{\psi(\cdot)} x(t)-x(b) \int_{0}^{1} \frac{\psi(\alpha)}{\Gamma(1-\alpha)}(b-t)^{-\alpha} d \alpha .
$$

Along the text, we use the notation

$$
\mathbb{I}_{b^{-}}^{1-\psi(\cdot)} x(t)=\int_{0}^{1} \psi(\alpha) \cdot I_{b^{-}}^{1-\alpha} x(t) d \alpha,
$$

where $I_{b^{-}}^{1-\alpha}$ represents the right Riemann-Liouville fractional integral of order $1-\alpha$.

The following results will be useful for our purposes. In concrete, integration by parts will be used in the proof of the Pontryagin maximum principle (Theorem 1).

Lemma 1 (Integration by parts formula [11]). Let $x \in L^{\alpha}$ and $y \in A C^{\alpha}$. Then,

$$
\int_{a}^{b} x(t) \cdot{ }^{C} \mathbb{D}_{a^{+}}^{\psi(\cdot)} y(t) d t=\left[y(t) \cdot \mathbb{I}_{b^{-}}^{1-\psi(\cdot)} x(t)\right]_{a}^{b}+\int_{a}^{b} y(t) \cdot \mathbb{D}_{b^{-}}^{\psi(\cdot)} x(t) d t .
$$

It follows a generalized Grönwall inequality that will be used in Section 3.1.

Lemma 2 (Grönwall inequality [16]). Let $\alpha$ be a positive real number and let $a(\cdot), b(\cdot)$, and $u(\cdot)$ be non-negative continuous functions on $[0, T]$ with $b(\cdot)$ monotonic increasing on $[0, T)$. If

$$
u(t) \leq a(t)+b(t) \int_{0}^{t}(t-s)^{\alpha-1} a(s) d s,
$$

then

$$
u(t) \leq a(t)+\int_{0}^{t}\left[\sum_{n=0}^{\infty} \frac{(b(t) \Gamma(\alpha))^{n}}{\Gamma(n \alpha)}(t-s)^{n \alpha-1}\right] d s
$$

for all $t \in[0, T)$.

\section{Main Results}

In this work, we look for an essentially bounded control $u \in L^{\infty}\left([a, b], \mathbb{R}^{m}\right)$ and the corresponding state trajectory $x \in A C^{\alpha}\left([a, b], \mathbb{R}^{n}\right)$, solution to the following distributedorder non-local fractional-order optimal control problem:

$$
\begin{aligned}
& J[x(\cdot), u(\cdot)]=\int_{a}^{b} L(t, x(t), u(t)) d t \longrightarrow \max , \\
& { }^{C} \mathbb{D}_{a^{+}}^{\psi(\cdot)} x(t)=f(t, x(t), u(t)), \quad t \in[a, b] \text { a.e., } \\
& x(\cdot) \in A C^{\alpha}, u(\cdot) \in L^{\infty}, \\
& x(a)=x_{a} \in \mathbb{R}^{n}, \quad u(t) \in \Omega,
\end{aligned}
$$

where $\Omega$ is a closed subset of $\mathbb{R}^{m}$. The data functions $L:[a, b] \times \mathbb{R}^{n} \times \mathbb{R}^{m} \rightarrow \mathbb{R}$ and $f:[a, b] \times \mathbb{R}^{n} \times \mathbb{R}^{m} \rightarrow \mathbb{R}^{n}$ are subject to the following assumptions:

- The function $f$ is continuous in all its three arguments. 
- The function $f$ is continuously differentiable with respect to state variable $x$ and, in particular, locally Lipschitz-continuous, that is, for every compact $B \subset \mathbb{R}^{n}$ and for all $x, y \in B$ there is $K>0$ such that

$$
\|f(t, x, u)-f(t, y, u)\| \leq K\|x-y\| .
$$

- With respect to the control $u$, there exists $M>0$ such that

$$
\|f(t, x, u)\| \leq M \quad \forall(t, x) \in[a, b] \times \mathbb{R}^{n} .
$$

- The cost integrand $L$ satisfies the same assumptions as $f$.

\subsection{Sensitivity Analysis}

Now, our concern is to establish continuity and differentiability results on the state solutions for any control perturbation (Lemmas 3-5), which are then used in Section 3.2 to prove a necessary optimality condition for the optimal control problem (1). With this purpose, let us denote by $\mathcal{L}[F(\cdot)]$ the set of all Lebesgue points in $[a, b)$ of the essentially bounded functions $t \mapsto f(t, x(t), u(t))$ and $t \mapsto L(t, x(t), u(t))$. Thus, let $(\tau, v) \in \mathcal{L}[F(\cdot)] \times$ $\Omega$, and, for every $\theta \in[0, b-\tau)$, let us consider the needle-like variation $u^{\theta} \in L^{\infty}\left([a, b], \mathbb{R}^{n}\right)$ associated to the optimal control $u^{*}$, which is given by

$$
u^{\theta}(t)= \begin{cases}u^{*}(t) & \text { if } \quad t \notin[\tau-\theta, \tau), \\ v & \text { if } \quad t \in[\tau-\theta, \tau),\end{cases}
$$

for almost every $t \in[a, b]$.

Lemma 3 (Continuity of solutions). For any $(\tau, v) \in \mathcal{L}[F(\cdot)] \times \Omega$, denote by $x^{\theta}$ the corresponding state trajectory to the needle-like variation $u^{\theta}$, that is, the state solution of

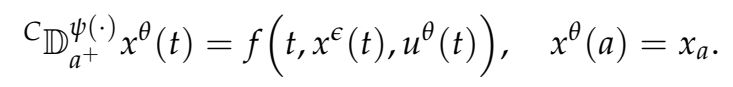

Then, we have that $x^{\theta}$ converges uniformly to the optimal state trajectory $x^{*}$ whenever $\theta$ tends to zero.

Proof. We have that

$$
\mathrm{C}_{\mathbb{D}_{a^{+}}^{\psi(\cdot)}}\left(x^{\theta}(t)-x^{*}(t)\right)=f\left(t, x^{\theta}(t), u^{\theta}(t)\right)-f\left(t, x^{*}(t), u^{*}(t)\right) .
$$

Then, by definition of the distributed-order operator,

$$
\int_{0}^{1} \psi(\alpha)^{C} D_{a^{+}}^{\alpha}\left(x^{\theta}(t)-x^{*}(t)\right) d \alpha=f\left(t, x^{\theta}(t), u^{\theta}(t)\right)-f\left(t, x^{*}(t), u^{*}(t)\right) .
$$

Now, using the mean value theorem for integrals, there exists an $\bar{\alpha}$ such that

$$
{ }^{C} D_{a^{+}}^{\bar{\alpha}}\left(x^{\theta}(t)-x^{*}(t)\right)=\frac{1}{m}\left[f\left(t, x^{\theta}(t), u^{\theta}(t)\right)-f\left(t, x^{*}(t), u^{*}(t)\right)\right]
$$

with

$$
m=\int_{0}^{1} \psi(\alpha) d \alpha
$$

Therefore, by the left inverse property, we obtain the following integral representation:

$$
x^{\theta}(t)-x^{*}(t)=\frac{1}{m} I_{a^{+}}^{\bar{\alpha}}\left(f\left(t, x^{\theta}(t), u^{\theta}(t)\right)-f\left(t, x^{*}(t), u^{*}(t)\right)\right) .
$$


Moreover, note that

$$
\begin{aligned}
f\left(t, x^{\theta}(t), u^{\theta}(t)\right)-f\left(t, x^{*}(t), u^{*}(t)\right)=\left\{f\left(t, x^{\theta}(t), u^{\theta}(t)\right)-f\left(t, x^{*}(t), u^{\theta}(t)\right)\right\} \\
+\left\{f\left(t, x^{*}(t), u^{\theta}(t)\right)-f\left(t, x^{*}(t), u^{*}(t)\right)\right\} .
\end{aligned}
$$

With the help of the triangular inequality, we can write that

$$
\begin{aligned}
\left\|x^{\theta}(t)-x^{*}(t)\right\| \leq \frac{1}{m} I_{a^{+}}^{\bar{\alpha}}(\| f( & \left.\left.t, x^{\theta}(t), u^{\theta}(t)\right)-f\left(t, x^{*}(t), u^{\theta}(t)\right) \|\right) \\
& +\frac{1}{m} I_{(\tau-\theta)^{+}}^{\bar{\alpha}}\left(\left\|f\left(t, x^{*}(t), u^{\theta}(t)\right)-f\left(t, x^{*}(t), u^{*}(t)\right)\right\|\right),
\end{aligned}
$$

since $u^{\theta}$ and $u^{*}$ are different only on $[\tau-\theta, \tau]$. From the Lipschitz property of $f$ and the boundedness with respect to the control, it follows that

$$
\left\|x^{\theta}(t)-x^{*}(t)\right\| \leq \frac{K}{m} I_{a^{+}}^{\bar{\alpha}}\left(\left\|x^{\theta}(t)-x^{*}(t)\right\|\right)+\frac{1}{m} \cdot 2 M \frac{\theta^{\bar{\alpha}}}{\Gamma(\alpha+1)} .
$$

Now, by applying the fractional Grönwall inequality (Lemma 2), it follows that

$$
\left\|x^{\theta}(t)-x^{*}(t)\right\| \leq \frac{2 M \theta^{\bar{\alpha}}}{m \Gamma(\alpha+1)}\left[1+\int_{a}^{t} \sum_{n=1}^{\infty} \frac{K^{n}}{\Gamma(n \bar{\alpha})}(t-s)^{n \bar{\alpha}-1} d s\right] \leq \omega_{1} \theta^{\bar{\alpha}},
$$

where $\omega_{1}=\frac{2 M}{m \Gamma(\alpha+1)} E_{\alpha, 1}\left(K(b-a)^{\alpha}\right)$, and $E_{\alpha, 1}$ is the Mittag-Leffler function of parameter $\bar{\alpha}$. Hence, by taking the limit when $\theta$ tends to zero, we obtain the desired result: $x^{\theta} \rightarrow x^{*}$ for all $t \in[a, b]$.

The next result is a corollary of Lemma 3.

Lemma 4. There exists $\omega_{2} \geq 0$ such that

$$
\left.\left.\left\|x^{\theta}(t)-x^{*}(t)\right\| \leq \omega_{2} \theta(t-(\tau-\theta))^{\bar{\alpha}-1} \quad \forall t \in\right] \tau-\theta, b\right] .
$$

Proof. Using similar arguments of Lipschitz-continuity of $f$ and its boundedness with respect to the control $u$, we get

$$
\begin{aligned}
& \left\|x^{\theta}(t)-x^{*}(t)\right\| \leq \frac{M}{m \Gamma(\bar{\alpha})} \int_{\tau-\theta}^{\tau}(t-s)^{\bar{\alpha}-1} d s \\
+ & \frac{K}{m \Gamma(\bar{\alpha})} \int_{\tau-\theta}^{\tau}(t-s)^{\bar{\alpha}-1}\left\|x^{\theta}(s)-x^{*}(s)\right\| d s+\frac{K}{m \Gamma(\bar{\alpha})} \int_{\tau}^{t}(t-s)^{\bar{\alpha}-1}\left\|x^{\theta}(s)-x^{*}(s)\right\| d s .
\end{aligned}
$$

Note that $\int_{\tau-\theta}^{\tau}(t-s)^{\bar{\alpha}-1} d s \leq \theta(t-(\tau-\theta))^{\bar{\alpha}-1}$, and, as a consequence of Lemma 3, we obtain that

$$
\begin{aligned}
\left\|x^{\theta}(t)-x^{*}(t)\right\| \leq \frac{M}{m \Gamma(\bar{\alpha})}\left(M+\omega_{1} K \theta^{\bar{\alpha}}\right) \theta & t-(\tau-\theta))^{\bar{\alpha}-1} \\
& +\frac{K}{m \Gamma(\bar{\alpha})} \int_{\tau}^{t}(t-s)^{\bar{\alpha}-1}\left\|x^{\theta}(s)-x^{*}(s)\right\| d s .
\end{aligned}
$$

We conclude the proof by applying again the fractional Grönwall inequality (Lemma 2), in which we set $\omega_{2}=\frac{1}{m} M+\omega_{1} K \theta^{\bar{\alpha}} E_{\alpha, 1}\left(K(b-a)^{\alpha}\right)$.

Lemma 5 (Differentiability of the perturbed trajectory). For all $(\tau, v) \in \mathcal{L}[F(\cdot)] \times \Omega$, we have that the variational trajectory $\frac{x^{\theta}(\cdot)-x^{*}(\cdot)}{\theta}$ is uniformly convergent to $\eta(\cdot)$ when $\theta$ tends 
to zero, where $\eta(\cdot)$ is the unique solution to the distributed-order left Caputo fractional Cauchy problem

$$
\left\{\begin{array}{l}
\left.\left.{ }_{\mathbb{D}_{\tau^{+}}}^{\psi(\cdot)} \eta(t)=\frac{\partial f\left(t, x^{*}(t), u^{*}(t)\right)}{\partial x} \cdot \eta(t), \quad t \in\right] \tau, b\right], \\
I_{\tau^{+}}^{1-\bar{\alpha}} \eta(\tau)=\frac{1}{m}\left[f\left(\tau, x^{*}(\tau), v\right)-f\left(\tau, x^{*}(\tau), u^{*}(\tau)\right)\right] .
\end{array}\right.
$$

Proof. Set $z^{\theta}(t)=\frac{x^{\theta}(t)-x^{*}(t)}{\theta}-\eta(t)$ for all $t \in[\tau, b]$. Our aim is to prove that $z^{\theta}$ converges uniformly to zero on $[\tau, b]$ whenever $\theta \rightarrow 0$. The integral representation of $z^{\theta}$ is given as follows:

$$
\begin{gathered}
z^{\theta}(t)=-\frac{1}{m \Gamma(\bar{\alpha})}(t-\tau)^{\bar{\alpha}-1}\left(f\left(\tau, x^{*}(\tau), v\right)-f\left(\tau, x^{*}(\tau), u^{*}(\tau)\right)\right) \\
+\frac{1}{m \Gamma(\bar{\alpha})} \int_{\tau^{+}}^{t}(t-s)^{\bar{\alpha}-1}\left[\frac{f\left(s, x^{\theta}(s), u^{*}(s)\right)-f\left(s, x^{*}(s), u^{*}(s)\right)}{\theta}\right. \\
\left.-\frac{\partial f\left(s, x^{*}(s), u^{*}(s)\right)}{\partial x} \times \frac{x^{\theta}(s)-x^{*}(s)}{\theta}\right] d s \\
+\frac{1}{m \Gamma(\bar{\alpha})} \int_{\tau^{+}}^{t}(t-s)^{\bar{\alpha}-1} \frac{\partial f\left(s, x^{*}(s), u^{*}(s)\right)}{\partial x} \times z^{\theta}(s) d s
\end{gathered}
$$

for every $t \in[\tau, b]$. Let us investigate the two first terms of the right-hand side of (4). By boundedness of $f$ with respect to $u$, we have that

$$
\left\|-\frac{1}{m \Gamma(\bar{\alpha})}(t-\tau)^{\bar{\alpha}-1}\left(f\left(\tau, x^{*}(\tau), v\right)-f\left(\tau, x^{*}(\tau), u^{*}(\tau)\right)\right)\right\| \leq \frac{2 M}{\Gamma(\bar{\alpha})}(b-\tau)^{\bar{\alpha}} .
$$

Further, using the classical Taylor formula with integral rest, we have

$$
\begin{array}{r}
\frac{f\left(s, x^{\theta}(s), u^{*}(s)\right)-f\left(s, x^{*}(s), u^{*}(s)\right)}{\theta}-\frac{\partial f\left(s, x^{*}(s), u^{*}(s)\right)}{\partial x} \times \frac{x^{\theta}(s)-x^{*}(s)}{\theta} \\
=\int_{0}^{1}\left(\frac{\partial f\left(s, x^{*}(s)+w\left(x^{\theta}(s)-x^{*}(s)\right), u^{*}(s)\right)}{\partial x}-\frac{\partial f\left(s, x^{*}(s), u^{*}(s)\right)}{\partial x}\right) \\
\times\left(\frac{x^{\theta}(s)-x^{*}(s)}{\theta}\right) d w .
\end{array}
$$

Hence, from Lemma 4, we deduce that $\left\|\frac{x^{\theta}(s)-x^{*}(s)}{\theta}\right\| \leq \omega_{2}(t-(\tau-\theta))^{\bar{\alpha}-1}$. Next, we set

$$
\varsigma_{\theta}(s)=\int_{0}^{1}\left\|\frac{\partial f\left(s, x^{*}(s)+w\left(x^{\theta}(s)-x^{*}(s)\right), u^{*}(s)\right)}{\partial x}-\frac{\partial f\left(s, x^{*}(s), u^{*}(s)\right)}{\partial x}\right\| d s,
$$

and, referring to Lemma A.3 in Reference [17], we get an estimate for the second term of (11), and we end the proof by application of the fractional Grönwall inequality of Lemma 2.

\subsection{Pontryagin's Maximum Principle of Distributed-Order}

It follows the main result of our work: a distributed-order Pontryagin maximum principle for the fractional-order optimal control problem (1).

Theorem 1 (Pontryagin Maximum Principle for (1)). If $\left(x^{*}(\cdot), u^{*}(\cdot)\right)$ is an optimal pair for (1), then there exists $\lambda \in L^{\alpha}$, called the adjoint function variable, such that the following conditions hold for all $t$ in the interval $[a, b]$ : 
- $\quad$ the maximality condition

$$
H\left(t, x^{*}(t), u^{*}(t), \lambda(t)\right)=\max _{\omega \in \Omega} H\left(t, x^{*}(t), \omega, \lambda(t)\right)
$$

- $\quad$ the adjoint system

$$
\mathbb{D}_{b^{-}}^{\psi(\cdot)} \lambda(t)=\frac{\partial H}{\partial x}\left(t, x^{*}(t), u^{*}(t), \lambda(t)\right) ;
$$

- the transversality condition

$$
\mathbb{I}_{b^{-}}^{1-\psi(\cdot)} \lambda(b)=0
$$

where the Hamiltonian $H$ is defined by

$$
H(t, x, u, \lambda)=L(t, x, u)+\lambda \cdot f(t, x, u) .
$$

Proof. First of all, note that the regularity of function $f$ with respect to the state variable (recall that $f$ is continuously differentiable with respect to $x$ ) is exactly as in our previous paper [12]. For this reason, the adjoint system (6) and its transversality condition (7) remain exactly the same as the ones proved in Reference [12]. Therefore, we only need to prove the maximality condition (5), which is new due to less regularity of $f$ with respect to control functions and the fact that now the controls take values on the closed $\Omega$ set. We start by using integration by parts (Lemma 1 ) for functions $\lambda \in L^{\alpha}$ and $\eta \in A C^{\alpha}$ on $[\tau, b]$ :

$$
\int_{\tau}^{b} \lambda(s) \cdot{ }^{C} \mathbb{D}_{\tau^{+}}^{\psi(\cdot)} \eta(s) d s=\left[\eta(s) \cdot \mathbb{I}_{b^{-}}^{1-\psi(\cdot)} \lambda(s)\right]_{\tau}^{b}+\int_{\tau}^{b} \eta(s) \cdot \mathbb{D}_{b^{-}}^{\psi(\cdot)} \lambda(s) d s,
$$

where $\lambda$ is the adjoint variable given in Reference [12]:

$$
\left\{\begin{array}{l}
\mathbb{D}_{b^{-}}^{\psi(\cdot)} \lambda(t)=\frac{\partial L}{\partial x}\left(t, x^{*}(t), u^{*}(t)\right)+\lambda(t) \cdot \frac{\partial f}{\partial x}\left(t, x^{*}(t), u^{*}(t)\right), \\
\mathbb{I}_{b^{-}}^{1-\psi(\cdot)} \lambda(b)=0 .
\end{array}\right.
$$

Substituting (9) and the variational differential system given in (3) into (8), we obtain that

$$
\begin{aligned}
\int_{\tau}^{b} \lambda(s) \cdot\left(\frac{\partial f\left(s, x^{*}(s), u^{*}(s)\right)}{\partial x} \cdot \eta(s)\right) d s=-\eta(\tau) \mathbb{I}_{b^{-}}^{1-\psi(\cdot)} \lambda(\tau) & \\
& +\int_{\tau}^{b} \eta(s)\left(\frac{\partial L}{\partial x}\left(s, x^{*}(s), u^{*}(s)\right)+\lambda(s) \cdot \frac{\partial f}{\partial x}\left(s, x^{*}(s), u^{*}(s)\right)\right) d s
\end{aligned}
$$

which leads to

$$
\eta(\tau) \mathbb{I}_{b^{-}}^{1-\psi(\cdot)} \lambda(\tau)=\int_{\tau}^{b} \eta(s) \cdot \frac{\partial L\left(s, x^{*}(s), u^{*}(s)\right)}{\partial x} d s .
$$

Next, recall that, from the definition of distributed-order fractional integral and the mean value theorem, we have the existence of an $\bar{\alpha}$ such that

$$
\mathbb{I}_{b^{-}}^{1-\psi(\cdot)} \lambda(\tau)=\int_{0}^{1} \psi(\alpha) I_{b^{-}}^{1-\bar{\alpha}} \lambda(\tau) d \alpha=m I_{b^{-}}^{1-\bar{\alpha}} \lambda(\tau)
$$

where $m=\int_{0}^{1} \psi(\alpha) d \alpha$. Moreover, by the fundamental law of calculus and the duality of the Riemann-Liouville integral operator, we have also that

$$
\begin{aligned}
\eta(\tau) I_{b^{-}}^{1-\bar{\alpha}} \lambda(\tau) & =\frac{d}{d \tau}\left(-\int_{\tau}^{b} \eta(s) I_{b^{-}}^{1-\bar{\alpha}} \lambda(s) d s\right) \\
& =\frac{d}{d \tau}\left(-\int_{\tau}^{b} \lambda(s) I_{\tau^{+}}^{1-\bar{\alpha}} \eta(s) d s\right) \\
& =\lambda(\tau) I_{\tau^{+}}^{1-\bar{\alpha}} \eta(\tau) .
\end{aligned}
$$


Next, using the boundary condition from system (3), it yields

$$
\begin{aligned}
\eta(\tau) \mathbb{I}_{b^{-}}^{1-\psi(\cdot)} \lambda(\tau) & =m \eta(\tau) I_{b^{-}}^{1-\bar{\alpha}} \lambda(\tau)=m \lambda(\tau) I_{\tau^{+}}^{1-\bar{\alpha}} \eta(\tau) \\
& =m \lambda(\tau)\left(\frac{1}{m}\left[f\left(\tau, x^{*}(\tau), v\right)-f\left(\tau, x^{*}(\tau), u^{*}(\tau)\right)\right]\right)
\end{aligned}
$$

that is, $\eta(\tau) \mathbb{I}_{b^{-}}^{1-\psi(\cdot)} \lambda(\tau)=\lambda(\tau) \cdot\left(f\left(\tau, x^{*}(\tau), v\right)-f\left(\tau, x^{*}(\tau), u^{*}(\tau)\right)\right)$. Finally, substituting this expression into (10), we get

$$
\lambda(\tau) \cdot\left(f\left(\tau, x^{*}(\tau), v\right)-f\left(\tau, x^{*}(\tau), u^{*}(\tau)\right)\right)=\int_{\tau}^{b} \eta(s) \cdot \frac{\partial L\left(s, x^{*}(s), u^{*}(s)\right)}{\partial x} d s .
$$

However, with respect to the cost functional $J$, the limit

$$
\lim _{\theta \rightarrow 0^{+}} \frac{J\left[x^{\theta}(\cdot), u^{\theta}(\cdot)\right]-J\left[x^{*}(\cdot), u^{*}(\cdot)\right]}{\theta} \leq 0
$$

because, by assumption, $\left(x^{*}, u^{*}\right)$ is an optimal pair. This limit can be written as

$$
\begin{aligned}
& \lim _{\theta \rightarrow 0^{+}} \frac{J\left[x^{\theta}(\cdot), u^{\theta}(\cdot)\right]-J\left[x^{*}(\cdot), u^{*}(\cdot)\right]}{\theta} \\
&=\lim _{\theta \rightarrow 0^{+}} \frac{1}{\theta} \int_{\tau-\theta}^{\tau}\left[L\left(s, x^{*}(s), v\right)-L\left(s, x^{*}(s), u^{*}(s)\right)\right] d s \\
& \quad+\lim _{\theta \rightarrow 0^{+}} \int_{\tau}^{b} \frac{L\left(s, x^{\theta}(s), u^{\theta}(s)\right)-L\left(s, x^{*}(s), u^{\theta}(s)\right)}{\theta} d s .
\end{aligned}
$$

Considering the fact that $\tau$ is a Lebesgue point of

$$
L\left(s, x^{*}(s), v\right)-L\left(s, x^{*}(s), u^{*}(s)\right):=\psi(s),
$$

it follows from the Lebesgue differentiation property

$$
\left|\frac{1}{\theta} \int_{\tau-\theta}^{\tau} \psi(s) d s-\psi(\tau)\right|=\left|\frac{1}{\theta} \int_{\tau-\theta}^{\tau}(\psi(s)-\psi(\tau)) d s\right| \leq \frac{1}{\theta} \int_{\tau-\theta}^{\tau}|\psi(s)-\psi(\tau)| d s
$$

that

$$
\begin{array}{r}
\lim _{\theta \rightarrow 0^{+}} \frac{1}{\theta} \int_{\tau-\theta}^{\tau}\left[L\left(s, x^{*}(s), v\right)-L\left(s, x^{*}(s), u^{*}(s)\right)\right] d s \\
=L\left(\tau, x^{*}(\tau), v\right)-L\left(\tau, x^{*}(\tau), u^{*}(\tau)\right) .
\end{array}
$$

Moreover, with respect to the third limit in (14), we can apply the Lipschitz property of $L$ to obtain

$$
\left|\frac{L\left(s, x^{\theta}(s), u^{\theta}(s)\right)-L\left(s, x^{*}(s), u^{\theta}(s)\right)}{\theta}\right| \leq K\left\|\frac{x^{\theta}-x^{*}}{\theta}\right\| .
$$

Therefore, because $\frac{x^{\theta}-x^{*}}{\theta}$ is a uniformly convergent series of functions, we conclude that the integrand

$$
\frac{L\left(s, x^{\theta}(s), u^{\theta}(s)\right)-L\left(s, x^{*}(s), u^{\theta}(s)\right)}{\theta}
$$

is uniformly bounded. Furthermore, we have

$$
L\left(s, x^{\theta}(s), u^{\theta}(t)\right)=L\left(s, x^{*}(s), u^{\theta}(s)\right)
$$




$$
+\left(x^{\theta}(s)-x^{*}(s)\right) \cdot \frac{\partial L\left(s, x^{*}(s), u^{\theta}(s)\right)}{\partial x}+o\left(\left\|x^{\theta}-x^{*}\right\|\right) .
$$

Next, by the continuity Lemma 3, we have $\left\|x^{\theta}-x^{*}\right\| \rightarrow 0$ whenever $\theta \rightarrow 0$. Thus, we can express the residue term only as function of $\theta$, that is,

$$
L\left(s, x^{\theta}(s), u^{\theta}(s)\right)=L\left(s, x^{*}(s), u^{\theta}(s)\right)+\left(x^{\theta}(s)-x^{*}(s)\right) \cdot \frac{\partial L\left(s, x^{*}(s), u^{\theta}(s)\right)}{\partial x}+o(\theta),
$$

and the following expression yields for the second limit:

$$
\begin{aligned}
\lim _{\theta \rightarrow 0} \frac{L\left(s, x^{\theta}(s), u^{*}(s)\right)-L\left(t, x^{*}(t), u^{*}(t)\right)}{\theta} & =\frac{\partial L\left(s, x^{*}(s), u^{*}(s)\right)}{\partial x} \cdot \lim _{\theta \rightarrow 0} \frac{\left(x^{\theta}(s)-x^{*}(s)\right)}{\theta} \\
& =\frac{\partial L\left(s, x^{*}(s), u^{*}(s)\right)}{\partial x} \cdot \eta(s) .
\end{aligned}
$$

Hence, thanks to the Lebesgue bounded convergence theorem,

$$
\lim _{\theta \rightarrow 0^{+}} \int_{\tau}^{b} \frac{L\left(s, x^{\theta}(s), u^{\theta}(s)\right)-L\left(s, x^{*}(s), u^{\theta}(s)\right)}{\theta} d s=\frac{\partial L\left(s, x^{*}(s), u^{*}(s)\right)}{\partial x} \cdot \eta(s)
$$

and, altogether, we get

$$
\begin{aligned}
& \lim _{\theta \rightarrow 0^{+}} \frac{J\left[x^{\theta}(\cdot), u^{\theta}(\cdot)\right]-J\left[x^{*}(\cdot), u^{*}(\cdot)\right]}{\theta} \\
&=L\left(\tau, x^{*}(\tau), v\right)-L\left(\tau, x^{*}(\tau), u^{*}(\tau)\right)+\frac{\partial L\left(s, x^{*}(s), u^{*}(s)\right)}{\partial x} \cdot \eta(s) .
\end{aligned}
$$

Hence, using inequality (13) and (12), we obtain that

$$
L\left(\tau, x^{*}(\tau), v\right)-L\left(\tau, x^{*}(\tau), u^{*}(\tau)\right)+\lambda(\tau) \cdot\left[f\left(\tau, x^{*}(\tau), v\right)-f\left(\tau, x^{*}(\tau), u^{*}(\tau)\right)\right] \leq 0,
$$

meaning that

$$
H\left(\tau, x^{*}(\tau), u^{*}(\tau), \lambda(\tau)\right) \geq H\left(\tau, x^{*}(\tau), v, \lambda(\tau)\right),
$$

where $H=L(t, x, u)+\lambda \cdot f(t, x, u)$. Because $\tau$ is an arbitrary Lebesgue point of the control $u^{*}$ and $v$ is an arbitrary element of the set $\Omega$, it follows that the relation

$$
H\left(t, x^{*}(t), u^{*}(t), \lambda(t)\right)=\max _{\omega \in \Omega} H\left(t, x^{*}(t), \omega, \lambda(t)\right)
$$

holds at all Lebesgue points, which ends the proof.

\section{An Illustrative Example}

As an example of application of our main result, let us consider the following distributed-order fractional optimal control problem:

$$
\begin{gathered}
J[x(\cdot), u(\cdot)]=\int_{1}^{5}(1-3 u(t)) x(t) d t \longrightarrow \max , \\
C_{\mathbb{D}_{1}}^{\psi(\cdot)} x(t)=u(t) x(t), \quad \text { a.e. } \quad t \in[1,5], \\
u(t) \in[0,2], \\
x(1)=x_{a}>0,
\end{gathered}
$$

where the distribution function of order of differentiation is given by

$$
\psi(\alpha)=\frac{\alpha}{3} .
$$


Let $u^{*}$ be an optimal control to problem (16). Theorem 1 give us a necessary optimality condition that $u^{*}$ must satisfy. The Hamiltonian function associated with this problem is given by

$$
H(t, x, u, \lambda)=(1-3 u) x+u x \lambda .
$$

From the maximality condition (5), we know that $u^{*}(t)$ maximizes a.e. in $[0,2]$ the mapping

$$
w \mapsto(1-3 w) x^{*}(t)+w x^{*}(t) \lambda(t) .
$$

Due to the positiveness of the initial condition $\left(x_{a}>0\right)$ and the linearity of the distributed order derivative, we have that $x^{*}(t)>0$ for all $t \in[1,5]$. Thus, the mapping to be maximized can be reduced to

$$
w \mapsto(\lambda(t)-3) w,
$$

and $u^{*}$ has the form

$$
u^{*}(t)= \begin{cases}2 & \text { if } \lambda(t)>3 \\ 0 & \text { if } \lambda(t)<3\end{cases}
$$

Now, it remains to determine the switching structure of the control through investigation of the adjoint boundary value problem given by (6) and (7), that is,

$$
\left\{\begin{array}{l}
\mathbb{D}_{5^{-}}^{\psi(\cdot)} \lambda(t)=1+(\lambda(t)-3) u^{*}(t), \\
\mathbb{I}_{b^{-}}^{1-\psi(\cdot)} \lambda(5)=0
\end{array}\right.
$$

Note that, because problem (16) does not have a terminal phase constraint, the fractional transversality condition (7) is simplified to $\lambda(5)=0$. Moreover, since $\lambda(\cdot)$ is a continuous function, there is $\xi>0$ such that $u^{*}(t)=0$ for all $t \in[5-\xi, 5]$. With this, we have that $\mathbb{D}_{5^{-}}^{\psi(\cdot)} \lambda(t)=1$, and it follows, by backward integration, that

$$
\lambda(t)=\frac{(5-t)^{\bar{\alpha}}}{m \Gamma(\bar{\alpha}+1)}
$$

where $m=\int_{0}^{1} \frac{\alpha}{3} d \alpha=\frac{1}{2}$ and $\bar{\alpha} \in[0,1]$. Noting that, for

$$
c:=5-(3 m \Gamma(\bar{\alpha}+1))^{\frac{1}{\bar{\alpha}}} \in[7 / 2,5[,
$$

we get $\lambda(c)=3$, we conclude that

$$
u^{*}(t)= \begin{cases}2 & \text { if } 0 \leq t<c \\ 0 & \text { if } c \leq t \leq 5\end{cases}
$$

\section{Conclusions}

Recent applications and experimental data-analysis studies have shown the importance of systems with "diffusing diffusivity" in anomalous diffusion, modeled with fractional, standard Brownian motions and distributed-order operators [18-20]. The theory of the calculus of variations for distributed-order fractional systems was initiated in 2018 by Almeida and Morgado [11], and it has been extended by the authors in 2020 to the more general framework of optimal control [12]. There, we established a weak Pontryagin Maximum Principle (PMP), under certain smoothness assumptions on the space of admissible functions, where the controls are not subject to any pointwise constraint [12]. The objective of the present article was to state and prove a strong version of the PMP for distributed-order fractional systems, valid for general non-linear dynamics and $L^{\infty}$ controls and, in contrast with References [11,12], without assuming that the controls take values on all the Euclidean space. Our statement is as general as possible, and it encompasses 
the distributed-order calculus of variations of [11] and the weak PMP of [12] as particular cases. Moreover, in the analysis of a strong version of PMP, we emphasized the use of needle-like variations to control perturbations, dealing with controls taking values on a closed set in a much larger class of admissible functions than in References [11,12]. Our approach began by proving results on continuity of solutions due to needlle-like variations, and then followed by a differentiability result on the state solutions with respect to perturbed trajectories. The statement and the proof of the Pontryagin Maximum Principle are rigorously given. Finally, the new necessary optimality conditions were illustrated by a simple example for which an analytical solution could be found. To deal with real optimal control problems of Nature, which are impossible to solve analytically, it is important to develop numerical methods based on the fractional distributed-order Pontryagin maximum principle here obtained. This will be subject of future research.

Author Contributions: The authors equally contributed to this paper, read and approved the final manuscript. Formal analysis, F.N. and D.F.M.T.; Investigation, F.N. and D.F.M.T.; Writing-original draft, F.N. and D.F.M.T.; Writing-review \& editing, F.N. and D.F.M.T. Both authors have read and agreed to the published version of the manuscript.

Funding: This research was funded by the Portuguese Foundation for Science and Technology (FCT), grant number UIDB/04106/2020 (CIDMA). Ndaïrou was also supported by FCT through the PhD fellowship PD/BD/150273/2019.

Data Availability Statement: Not applicable.

Acknowledgments: The authors are grateful to three anonymous reviewers for several valuable comments, which helped them to improve the manuscript.

Conflicts of Interest: The authors declare no conflict of interest. The funders had no role in the design of the study; in the collection, analyses, or interpretation of data; in the writing of the manuscript, or in the decision to publish the results.

\section{References}

1. Caputo, M. Mean fractional-order-derivatives differential equations and filters. Ann. Univ. Ferrara Sez. VII 1995, $41,73-84$.

2. Chechkin, A.V.; Gorenflo, R.; Sokolov, I.M. Retarding subdiffusion and accelerating superdiffusion governed by distributed-order fractional diffusion equations. Phys. Rev. E 2002, 66, 046129. [CrossRef] [PubMed]

3. Naber, M. Distributed order fractional sub-diffusion. Fractals 2004, 12, 23. [CrossRef]

4. Kochubei, A.N. Distributed order calculus and equations of ultraslow diffusion. J. Math. Anal. Appl. 2008, 340, 257-281. [CrossRef]

5. Mainardi, F.; Mura, A.; Pagnini, G. Time-fractional diffusion of distributed order. J. Vib. Control 2008, 14, 1267-1290. [CrossRef]

6. Vieira, N.; Rodrigues, M.M.; Ferreira, M. Time-fractional telegraph equation of distributed order in higher dimensions. Commun. Nonlinear Sci. Numer. Simul. 2021, 102, 105925. [CrossRef]

7. Kumar, Y.; Singh, V.K. Computational approach based on wavelets for financial mathematical model governed by distributed order fractional differential equation. Math. Comput. Simul. 2021, 190, 531-569. [CrossRef]

8. Ding, W.; Patnaik, S.; Sidhardh, S.; Semperlotti, F. Applications of Distributed-Order Fractional Operators: A Review. Entropy 2021, 23, 110. [CrossRef] [PubMed]

9. Abdelkawy, M.A. Numerical solutions for fractional initial value problems of distributed-order. Intern. J. Mod. Phys. C 2021, 32, 2150096. [CrossRef]

10. Almeida, R.; Torres, D.F.M. A survey on fractional variational calculus. In Handbook of Fractional Calculus with Applications; De Gruyter: Berlin, Germany, 2019; Volume 1, pp. 347-360.

11. Almeida, R.; Morgado, M.L. The Euler-Lagrange and Legendre equations for functionals involving distributed-order fractional derivatives. Appl. Math. Comput. 2018, 331, 394-403. [CrossRef]

12. Ndaïrou, F.; Torres, D.F.M. Distributed-Order Non-Local Optimal Control. Axioms 2020, 9, 124. [CrossRef]

13. Almeida, R.; Pooseh, S.; Torres, D.F.M. Computational Methods in the Fractional Calculus of Variations; Imperial College Press: London, UK, 2015. [CrossRef]

14. Samko, S.G.; Kilbas, A.A.; Marichev, O.I. Fractional Integrals and Derivatives; Gordon and Breach Science Publishers: Yverdon, Switzerland, 1993.

15. Caputo, M. Elasticità e Dissipazione; Zanichelli: Bologna, Italy, 1969.

16. Ye, H.; Gao, J.; Ding, Y. A generalized Gronwall inequality and its application to a fractional differential equation. J. Math. Anal. Appl. 2007, 328, 1075-1081. [CrossRef]

17. Bergounioux, M.; Bourdin, L. Pontryagin maximum principle for general Caputo fractional optimal control problems with Bolza cost and terminal constraints. ESAIM Control Optim. Calc. Var. 2019, 26, 35. [CrossRef] 
18. Golan, Y.; Sherman, E. Resolving mixed mechanisms of protein subdiffusion at the T cell plasma membrane. Nat. Commun. 2017, 8, 15851. [CrossRef] [PubMed]

19. Cherstvy, A. G.; Thapa, S.; Wagner, C. E.; Metzler, R. Non-Gaussian, non-ergodic, and non-Fickian diffusion of tracers in mucin hydrogels. Soft Matter 2019, 15, 2526-2551. [CrossRef] [PubMed]

20. Korabel, N.; Han, D.; Taloni, A.; Pagnini, G.; Fedotov, S.; Allan, V.; Waigh, T.A. Local analysis of heterogeneous intracellular transport: Slow and fast moving endosomes. Entropy 2021, 23, 958. [CrossRef] 